\title{
Transport in amorphous solid water films: Implications for self-diffusivity
}

\author{
Sean M. McClure, Evan T. Barlow, Minta C. Akin, Douglas J. Safarik,
} Thomas M. Truskett, and C. Buddie Mullins

Online Supplementary Material

\section{$\mathrm{CCl}_{4}$ Barrier Layer Effectiveness: Methanol Probe Experiments}

Determining the effectiveness of the $\mathrm{CCl}_{4}$ barrier layers employed in our structured films (such as those in Figures 2 and 3) is important with regard to the interpretation and conclusions drawn from such TPD mixing experiments. Intuitively, one would expect the presence hydrophobic, immiscible $\mathrm{CCl}_{4}$ barrier layer which covers the entire $\mathrm{H}_{2}{ }^{16} \mathrm{O} / \mathrm{H}_{2}{ }^{18} \mathrm{O}$ interface to hinder bulk diffusion between isotopically labeled ASW layers until the onset of crystallization-induced cracks (molecular volcano) ${ }^{65}$, the point at which the $\mathrm{CCl}_{4}$ diffusion barrier layer is allowed to escape from beneath the water overlayer. The hydrophobic effects of the $\mathrm{CCl}_{4}$ layer and the additional spacing between the films ( $\left.t \sim \mathrm{L}^{2} / \mathrm{D}\right)$ are both factors that should contribute to this hindering effect. However, issues such as $\mathrm{CCl}_{4}$ barrier layer roughness and $\mathrm{CCl}_{4}$ wetability (how "well" $\mathrm{CCl}_{4}$ covers the entire $\mathrm{H}_{2}{ }^{16} \mathrm{O} / \mathrm{H}_{2}{ }^{18} \mathrm{O}$ interface) could influence the effectiveness of the $\mathrm{CCl}_{4}$ barrier toward blocking $\mathrm{H}_{2} \mathrm{O}$ bulk diffusion, and thus could have important consequences for the validity of our main conclusions regarding ASW self-diffusivity. Hence, in this supplementary section, we have attempted to test the effectiveness of the $\mathrm{CCl}_{4}$ diffusion barrier layer using methanol $(\mathrm{MeOH})$ as a barrier "probe" molecule (Figures S3 and S4). Consistent with previous studies ${ }^{28}$, we find that the polar $\mathrm{MeOH}$ molecule exhibits mobility through dense ASW films. TPD-TOF SIMS experiments by 
Souda ${ }^{28}$ and TPD experiments by Livingston et. al. ${ }^{94}$ have also confirmed that $\mathrm{MeOH}$ appears to be, at low temperatures, fairly mobile in thin amorphous ${ }^{28}$ and $\mathrm{CI}^{94}$ films (although, in contrast to the results of Souda et. al. ${ }^{28}$ we do not see a suppression of morphological change in ASW due to the $\mathrm{MeOH}$ 'pill'). Since $\mathrm{MeOH}$ exhibits mobility in nanoscale ASW films, it can potentially be used to investigate the effectiveness of $\mathrm{CCl}_{4}$ layers in hindering bulk diffusion of $\mathrm{MeOH}$. If $\mathrm{CCl}_{4}$ provides a good barrier to $\mathrm{MeOH}$ mobility, one might expect it to be a good diffusion barrier for $\mathrm{H}_{2} \mathrm{O}$.

Figures S3(a)-(e) shows desorption of multilayers of $\mathrm{MeOH}$ from bare $\operatorname{Ir}(111), 30$ BL ASW $\mathrm{H}_{2} \mathrm{O}$, and from underneath various water overlayers (15-90 BL). $\mathrm{MeOH}$ multilayer desorption from the $\operatorname{Ir}(111)$ substrate (Figure S3(a)) appears to occur between $\mathrm{T} \sim 125-143 \mathrm{~K}$ for a MeOH coverage of $\sim 8 \mathrm{ML}$, with a desorption activation energy of $\mathrm{E}_{\mathrm{d}}$ $\sim 0.4 \mathrm{eV}$, based on zero order desorption kinetics. When $\mathrm{MeOH}$ multilayers are deposited and desorbed from atop a $30 \mathrm{BL} \mathrm{H}_{2}{ }^{16} \mathrm{O}$ ASW film, two desorption features become apparent in the subsequent TPD spectra, as shown in Figure S3(b). In this experiment, $30 \mathrm{BL} \mathrm{H}_{2}{ }^{16} \mathrm{O}$ ASW has been deposited first, followed by dosing of $\sim 8 \mathrm{ML}$ $\mathrm{MeOH}$. Upon heating, the first desorption feature occurs near the $\mathrm{MeOH}$ multilayer desorption feature ( $\mathrm{T} \sim 130-145 \mathrm{~K}$ ), with the second occurring near the end of the $\mathrm{H}_{2} \mathrm{O}$ desorption feature. This second desorption feature, occurring between T 160 K-170 K, is coincident with the end of desorption of the underlying water film. Figures S3(c)-(e) show TPD spectra from samples in which $\sim 8 \mathrm{ML}$ MeOH has been dosed on the $\operatorname{Ir}(111)$ sample first, followed by dosing of ASW overlayers of varying thickness (30-90 BL). As the spectra illustrate, the first $\mathrm{MeOH}$ desorption feature shifts in temperature as a function of water overlayer thickness, from T $\sim 143 \mathrm{~K}$ [Fig. S3(c)] to T $~ 152 \mathrm{~K}$ [Fig. S3(e)], 
presumably due to the additional ASW layers the methanol must traverse to reach the surface of the ASW film. Interestingly, no "volcano" effect is seen for MeOH desorption during ASW crystallization ( $\mathrm{T} \sim 155 \mathrm{~K}$ ), suggesting that $\mathrm{MeOH}$ is able to intermix well with the ASW film prior to crystallization and film fracture. After its initial desorption feature, $\mathrm{MeOH}$ proceeds to desorb from each of the films in Figs. S3(c)-(e) as the ASW film crystallizes and desorbs from the sample. A second $\mathrm{MeOH}$ feature again is seen concurrent with the completion of desorption of the water overlayer, increasing in intensity with increasing ASW overlayer thickness. While currently we can only speculate on the nature of this second TPD feature, it could be due to an associated water/methanol complex (such as a methanol hydrate) which is known to occur in low temperature $\mathrm{H}_{2} \mathrm{O} / \mathrm{MeOH}$ mixtures with high methanol concentration ( 1:1 ratio). ${ }^{95}$ This may explain why, even when $\mathrm{MeOH}$ is placed atop the ASW film, it still exhibits a $\mathrm{MeOH}$ feature coincident with the completion of ASW desorption. Our primary focus here lies in the ability of the $\mathrm{MeOH}$ (as demonstrated by Figs. S3(a)-(e)) to exhibit mobility through dense ASW films on these length scales prior to crystallization (and, hence, prior to porosity formation). As we will attempt to illustrate in the following discussion, this information can be used to help study the effectiveness of $\mathrm{CCl}_{4}$ barrier layers to $\mathrm{MeOH}$ transport in structured ASW films.

Figures S4(a)-(e) show TPD spectra obtained from structured films of $\mathrm{MeOH}$, $\mathrm{CCl}_{4}$, and $\mathrm{H}_{2}{ }^{16} \mathrm{O}$ ASW (see figure schematic) with various $\mathrm{CCl}_{4}$ barrier layer thicknesses (0 - $60 \mathrm{ML}$ ). These films were constructed in the following manner: first, $8 \mathrm{ML} \mathrm{MeOH}$ are dosed onto the $\operatorname{Ir}(111)$ sample; next $30 \mathrm{BL} \mathrm{H}_{2}{ }^{16} \mathrm{O}$ ASW are dosed; third, a $\mathrm{CCl}_{4}$ barrier layer of desired thickness was dosed; finally, $30 \mathrm{BL} \mathrm{H}_{2}{ }^{16} \mathrm{O}$ ASW are dosed and 
the sample was heated at $0.6 \mathrm{~K} / \mathrm{s}$. The spectra show that when $\mathrm{CCl}_{4}$ barrier layers are present in ASW films, the $\mathrm{MeOH}$ desorption trace is delayed, presumably due to the presence (hydrophobicity and additional length) of the $\mathrm{CCl}_{4}$ barrier layer. While it appears that $5 \mathrm{ML} \mathrm{CCl}_{4}$ [Fig. S4(b)] provides some hindrance to $\mathrm{MeOH}$ transport through the structured film (onset of MeOH desorption is delayed from T 148 K until T 152 K), $15 \mathrm{ML}_{\text {of }} \mathrm{CCl}_{4}$ [Fig. S4(c)] appears to be a sufficient thickness to completely halt $\mathrm{MeOH}$ desorption until the molecular volcano feature ( $\mathrm{T} \sim 155 \mathrm{~K}$ ). For $\mathrm{CCl}_{4}$ barrier thicknesses of 15 - 60 ML [Figs. S4(c)-(e)], the first MeOH desorption feature is delayed and always appears concurrent with the molecular volcano feature, the point at which the $\mathrm{CCl}_{4}$ diffusion barrier layer is allowed to escape. Experiments conducted at TPD ramp rates of $\beta=0.05 \mathrm{~K} / \mathrm{s}$ and $\beta=2 \mathrm{~K} / \mathrm{s}$ show similar behavior; i.e. the appearance of $\mathrm{MeOH}$ tracer occurs concurrent with abrupt desorption of $\mathrm{CCl}_{4}$ due to crystallization induced porosity.

We interpret the results of Figures S3 and S4 to suggest that $\mathrm{CCl}_{4}$ barrier layers with thicknesses greater than $15 \mathrm{ML}$ can provide an effective barrier to $\mathrm{MeOH}$ transport prior the onset of film fracture. Though $\mathrm{CCl}_{4}$ barrier layers with a thickness of less than 15 ML do provide some hindrance to $\mathrm{MeOH}$ transport, it is possible that these thicknesses do not provide a complete conformal layer between the ASW films. We speculate this behavior could arise due to de-wetting of the initial layers of $\mathrm{CCl}_{4}$ on ASW due to hydrophobic effects. In light of these results, it seems reasonable to expect that bulk transport of $\mathrm{H}_{2} \mathrm{O}$, a molecule similar to $\mathrm{MeOH}$, would also be hindered by the presence of the $\mathrm{CCl}_{4}$ layer, due to both the hydrophobicity and additional length of the $\mathrm{CCl}_{4}$ layer. 
One point of our discussion of Figures S3 and S4, worth mentioning, remains a bit ambiguous. Methanol, $(\mathrm{MeOH})$, is a molecule similar to $\mathrm{H}_{2} \mathrm{O}$ in many ways (i.e., polar, hydrogen-bonding, highly miscible in water) Thus, it seems $\mathrm{MeOH}$ might be a good 'probe' molecule to test the effectiveness of the non-polar, $\mathrm{CCl}_{4}$ 'barrier' layers to $\mathrm{H}_{2} \mathrm{O}$ mobility. Results displayed in Figure S4 seem to suggest that these layers do hinder $\mathrm{MeOH}$ mobility (appearance of $\mathrm{MeOH}$ desorption) in structured ASW and $\mathrm{CCl}_{4}$ films. One question that naturally arises is, if $\mathrm{MeOH}$ which can intermix well with $\mathrm{H}_{2} \mathrm{O}$, why shouldn't water exhibit similar diffusive mobility in thin ASW films near the same temperatures. Though we do not have a simple answer to this issue at this time, it should be reiterated that the evidence of low temperature $\mathrm{MeOH}$ mobility in low temperature $\mathrm{H}_{2} \mathrm{O}$ phases is not unique to our study. As mentioned earlier, previous investigations have suggested that $\mathrm{MeOH}$ is mobile in both $\mathrm{ASW}^{28}$ and $\mathrm{CI}^{94}$ thin films. Experiments by Souda et. al. ${ }^{28}$ (employing thin ASW and $\mathrm{MeOH}$ films) suggest that $\mathrm{MeOH}$ intermixes with ASW around T 135-140 K. This apparent mobility has indeed been $\operatorname{cited}^{28}$ as evidence of a glass-liquid transition in thin ASW films. Despite these ambiguities, we believe the $\mathrm{MeOH}$ experiments of Figures S3 and S4 are instructive in examining the effectiveness of the $\mathrm{CCl}_{4}$ layers in hindering bulk diffusive transport and do provide some useful information with regards to the present study. We view these experiments as an opportunity to probe the effectiveness of the $\mathrm{CCl}_{4}$ layer, using thermal desorption techniques, with a molecule that appears to exhibit mobility across the ASW film prior to crystallization (Figure S3). This "high" mobility of $\mathrm{MeOH}$ in ASW allows for investigation of the effectiveness of the $\mathrm{CCl}_{4}$ layer prior to film crystallization with a molecule "similar" to $\mathrm{H}_{2} \mathrm{O}$. As illustrated by Figure S4, at thicknesses greater than 15 
$\mathrm{ML}$, the $\mathrm{CCl}_{4}$ 'barrier' layer appears to block the appearance of $\mathrm{MeOH}$ at the film surface prior to film fracture.

These results provide some additional support for the idea that $\mathrm{CCl}_{4}$ layers should hinder interlayer bulk diffusion in structured, labeled ASW films (Figures 2 and 3). This suggests the substantial $\mathrm{H}_{2} \mathrm{O}$ self-transport exhibited by these films during crystallization is not due to bulk diffusion; rather, we argue the mixing to be primarily attributed to cracking/fracturing of the crystallizing ASW films. 